\title{
Complementary Study of Collagen State in Bladder Diseases Using Cross-Polarization Optical Coherence Tomography, Nonlinear and Atomic Force Microscopy
}

\author{
DOI: 10.17691/stm2017.9.1.01
}

Received November 24, 2016

E.B. Kiseleva, PhD, Researcher, Laboratory of Biotissue Optical Structure, Institute of Biomedical Technologies';

E.V. Gubarkova, PhD, Junior Researcher, Laboratory of Biotissue Optical Structure, Institute of Biomedical Technologies";

V.V. Dudenkova, Junior Researcher, Laboratory of Biotissue Optical Structure, Institute of Biomedical Technologies'; PhD Student, Faculty of Radiophysics;

P.S. Timashev, DSc, Head of the Novel Biomaterial Unit3; Senior Researcher, Laboratory of Laser Chemistry ${ }^{4}$;

S.L. Kotova, PhD, Senior Researcher, Laboratory of Modified Polymer Systems ${ }^{5}$; Leading Researcher, Department of Modern Biomaterials";

L.B. Timofeeva, PhD, Assistant, Department of Histology with Cytology and Embriology';

M.Yu. Kirillin, PhD, Senior Researcher, Laboratory of Biophotonics;

G.V. Belkova, PhD, Senior Researcher, Laboratory of Modified Polymer Systems ${ }^{5}$;

A.B. Solov'eva, DSc, Head of the Laboratory of Modified Polymer Systems ${ }^{5}$;

O.S. Strel'tsova, MD, DSc, Professor, Department of Urology named after E.V. Shahov';

N.D. Gladkova, MD, DSc, Professor, Vice Director for Science, Institute of Biomedical Technologies ${ }^{1}$

${ }^{1}$ Nizhny Novgorod State Medical Academy, 10/1 Minin and Pozharsky Square, Nizhny Novgorod, 603005,

Russian Federation;

2Lobachevsky State University of Nizhni Novgorod, 23 Prospect Gagarina, Nizhny Novgorod, 603950,

Russian Federation;

3I.M. Sechenov First Moscow State Medical University, 8/2 Trubetskaya St., Moscow, 119991,

Russian Federation;

${ }^{4}$ Institute of Photon Technologies, Federal Scientific Research Center "Crystallography and Photonics"

of the Russian Academy of Sciences, 2 Pionerskaya St., Moscow, Troitsk, 142190, Russian Federation;

${ }^{5}$ Semenov Institute of Chemical Physics of the Russian Academy of Sciences, 4 Kosygina St., Moscow, 119991,

Russian Federation;

${ }^{6}$ Institute of Applied Physics of the Russian Academy of Sciences, 46 Ul'yanova St., Nizhny Novgorod, 603950,

Russian Federation

The aim of the study is to demonstrate the potential of cross-polarization optical coherence tomography (CP OCT) as a minimally invasive real-time technique for detection of bladder cancer against a background of severe inflammation.

Materials and Methods. For the verification of CP OCT diagnostic data related to the condition of collagen comparison with highresolution microscopy inspection was performed and correlation of the results was calculated. The CP OCT study was performed on samples of tissue with muscle-invasive urothelial carcinoma $T_{2-3} N_{0-x} M_{0}(G 2,3)$ in 18 patients ranging in age from 50 to 64 obtained in course of cystectomy with urine diversion into the intestinal vessel. In total, 60 regions of interest were selected and divided into four groups in accordance with the results of the histological analysis: areas with mild inflammation (group 1, control), 12 areas; areas with severe inflammation (group 2), 18 areas; areas with poorly differentiated urothelial carcinoma with invasion into the muscular layer (group 3), 24 areas; areas with cancer recurrence at the post-operative scar (group 4), 6 areas. Tissue changes at micro-structural level registered by CP OCT were investigated in detail with high-resolution microscopy (nonlinear microscopy and atomic force microscopy). Quantitative processing of all the obtained images enabled their direct comparison.

For contacts: Elena B. Kiseleva, e-mail: kiseleva84@gmail.com 
Results. By two high-resolution techniques and classical histology the spatial and structural features of the bladder extracellular matrix organization exhibiting different pathologies were demonstrated. Depolarization factor maps derived from CP OCT images of both invasive carcinoma and carcinoma recurrence in the area of post-operative scar tissue characterize the cross-scattering properties of the bladder connective tissue in these conditions and demonstrate a statistically significant difference in the integral depolarization factor value for carcinoma and severe chronic inflammation $(p<0.05)$. A high statistically significant positive correlation $(r=0.867, p<0.001$; Spearman correlation) between the integral depolarization factor and the conformance parameter of the second harmonic signal distribution detected from collagen at the level of the mucous membrane was demonstrated. This is indicative of a high level of correspondence between the level of cross-scattering and the collagen fibers ordering. In cases of severe inflammation, invasive growth of urothelial carcinoma, and cancer recurrence in the area of the surgical scar the structural disorganization of fiber structures determined at tissue level in the subsurface region of the bladder was revealed.

Conclusion. The comparison of CP OCT images with data from the high-resolution techniques (nonlinear microscopy in second harmonic generation mode and atomic force microscopy) demonstrated that CP OCT have the potential to become a powerful, minimally invasive, real-time tool for detection of bladder cancer against a background of severe inflammation in the area of the post-operative scar. These results will stimulate further development of CP OCT technique and promote its active introduction into clinical practice.

Key words: cross-polarization optical coherence tomography; СP OCT; urothelial carcinoma; urothelial carcinoma recurrence; collagen state; nonlinear microscopy; second harmonic generation; atomic force microscopy.

Improvement of real-time in vivo techniques for diagnostics, in particular, for monitoring of cancer invasion, based on assessment of microstructural changes, remains one of the major directions for the development of endoscopic imaging techniques [1, 2].

Optical coherence tomography (OCT) technique in urology is primarily used for the identification and differential diagnosis of urothelial carcinoma, where quantitative processing of the OCT signal allows objectification of the changes observed. Quantitative processing of 3D OCT images was demonstrated to significantly increase the diagnostic performance of the method for in situ detection of bladder carcinomas [35]. Meanwhile, an analysis of a large number of works on the use of OCT for bladder cancer visualization by Hale and Deem [6] indicated that cases of false positive cancer diagnoses are fairly typical for OCT against a background of severe inflammation as well as in cases of the diagnosis of cancer in the region of the postoperative scar.

Polarization-sensitive OCT is an OCT modality that allows assessment of the condition of collagen in the region of interest (ROI) and is potentially able to increase the efficiency of bladder cancer detection [7, 8]. In our previous study [9], cross-polarization OCT (CP OCT), a modality of polarization-sensitive OCT, was demonstrated to evaluate the condition of the extracellular matrix of the bladder wall according to the cross-polarization backscattering. The degree and nature of the cross-scattering are mainly determined by the condition of the collagen fibers, and depends on the severity of inflammation and/or the stage of cancer development. According to our data, the effectiveness of CP OCT diagnostics in detection of superficial bladder cancer by inspecting cross-scattering properties of the mucous membrane is higher than that with traditional OCT: the diagnostic accuracy of CP OCT was $93.6 \%$, sensitivity was $96.4 \%$, specificity was $92.1 \%$, against $72.1,74.5$ and $70.8 \%$ for traditional OCT, respectively.
The development of high resolution methods of microscopy provided new abilities to study the changes in the spatial organization of the extracellular matrix during the course of inflammation and cancer invasion [10-13]. The method of nonlinear microscopy in second harmonic generation (SHG) mode allows imaging of collagen fibers in their normal condition and observation of the changes that occur during the process of inflammation and the development of neoplasia [10, 14]. However, this method of microscopy has serious limitations for in vivo endoscopic use [12, 15].

Atomic force microscopy (AFM) is considered to be promising for diagnoses of a variety of pathologies including connective tissues diseases and cancer $[16,17]$. By means of $3 D$ visualization at nanometer resolution of the surface profile of the objects under study (which is superior to the resolution of optical microscopy [18]), the AFM method is able to significantly complement and extend the knowledge of the mechanisms of pathology development at tissue (macro-) level.

In this study we performed a comparison of values obtained from the quantitative processing of images of the bladder mucous and submucous membranes, obtained using the CP OCT technique, with nonlinear microscopy and AFM in cases of chronic inflammation and the development of urothelial carcinoma against this background. Chosen combination of methods provided the possibility of tracing the condition of collagen at different levels of its organization: at the tissue level (change of cross-scattering in the $\mathrm{CP}$ OCT images), at the fiber level (change of geometrical distribution of the fibers in the SHG images) and at the fibrillar level (defects in collagen fibril packing in the AFM images).

The aim of the study is to demonstrate the potential of CP OCT as a minimally invasive method operating in real-time for the diagnosis of bladder cancer against a background of severe inflammation by means of 
verification of the obtained diagnostic data through comparison and calculation of the correlation with quantitative characterization of the collagen state based on high resolution microscopy inspection.

\section{Materials and Methods}

Human bladder wall post-surgery samples. The CP OCT study was performed with tissue samples obtained in the course of cystectomy with urine diversion to the intestinal vessel, in cases of muscle-invasive urothelial carcinoma $T_{2-3} N_{0-x} M_{0}(G 2,3)$ from 18 patients ranging in age from 50 to 64 . In total 60 areas of interest were selected. These were divided into four groups in accordance with the results of histological assessments: areas with mild inflammation (group 1, control), 12 areas; areas with severe inflammation (group 2), 18 areas; areas with poorly differentiated urothelial carcinoma with invasion into the muscular layer (group 3), 24 areas; and areas with carcinoma recurrence in the area of the postoperative scar (group 4), 6 areas. Due to inspection of materials from excised organs sourced from cases of muscle-invasive urothelial carcinoma developing against a background of long-term chronic inflammation (the duration of chronic inflammation was, on average, $17 \pm 6$ years), no areas of unaltered mucous membrane were found. In this respect, samples with mild inflammation (group 1) with minimal changes of the connective tissues were used as the control group.

Post-operative material was placed in a gauze moistened with phosphate buffer at $7^{\circ} \mathrm{C}$ and delivered for examination within $2 \mathrm{~h}$. After the CP OCT imaging, the OCT inspection area on the sample surface was marked with an ink, and serially sliced in the central area. Ethics Committee of the Nizhny Novgorod State Medical Academy, report No.7 dated August 14, 2014, for scientific research involving ex vivo human samples approved the clinical study.

Cross-polarization optical coherence tomography. A spectral modification of the CP OCT device with a common optical path for the reference and signal waves was used in this study. Such a modification provides real-time visualization of the backscattering maps from the tissue optical inhomogeneities. This device has two channels: a co-polarization channel and a cross-polarization one allowing the separation of the light waves into those (after backscattering in the tissue) preserving their initial state of polarization, or have been transformed to an orthogonal one. As a result, a CP OCT image displayed on the PC screen consists of two images: one in co- and the other in cross-polarization [19-21]. Each image represents a $4 \times 2 \mathrm{~mm}$ (width $\times$ height) area. The device uses probing radiation at a wavelength of $1,310 \mathrm{~nm}$, with a spectrum width of $100 \mathrm{~nm}$ and a power at the object of $20 \mathrm{~mW}$. The in-depth resolution (in air) is $\sim 10 \mu \mathrm{m}$, and the lateral resolution is $\sim 15 \mu \mathrm{m}$, while the imaging depth in air is $\sim 1.7 \mathrm{~mm}$, with a scanning rate of $20,000 \mathrm{~A}$-scans/s resulting in B-scans acquisition rate of 40 frames/s.

Quantitative assessment of the bladder tissue CP
OCT images was performed by generating depolarization factor maps and calculation of the integral depolarization factor (IDF). Each map represents a $2 \times 1 \mathrm{~mm}$ (width $\times$ height) area including the main ROI. The algorithm for calculating the depolarization factor had previously been developed by our group for assessing the condition of collagen fibers in different tissues (bladder and aorta mucous membranes) [22, 23]. The IDF calculation is based on the ratio of the OCT signal levels in the crossand co-polarization channels, averaged over the ROI.

Histological examination. After obtaining the $\mathrm{CP}$ OCT images, the bladder wall samples were fixed in $10 \%$ neutral formalin solution within $48 \mathrm{~h}$, then embedded in paraffin. Serial sections with a thickness of $7 \mu \mathrm{m}$ were prepared from the marked area; a number of sections were stained with hematoxylin and eosin and by Van Gieson method, while the others (after de-embedding) were inspected using SHG microscopy and AFM. The histological specimens were examined using a Leica DM2500 microscope (Leica Microsystems, Germany), equipped with a DFC $245 \mathrm{C}$ digital camera.

Nonlinear microscopy. This study was performed with an LSM 710 multiphoton laser scanning microscope (Carl Zeiss, Germany). The excitation was performed by the radiation of a Ti:Sapphire femtosecond Chameleon Vision II laser (Coherent, USA) with a pulse repetition rate of $\sim 80 \mathrm{MHz}$ and a duration of $140 \pm 20 \mathrm{fs}$. Deembedded 7-micron-thick sections of the bladder samples were observed at $170 \mu \mathrm{m}$-thick cover glass. Images of a fine structure were generated by means of a C-Apochromat water-immersion objective with a $40 \times$ zoom range and a numerical aperture of 1.2 , which provided a field of view of $212 \times 212 \mu \mathrm{m}$ with a resolution of $1,024 \times 1,024$ pixels.

The excitation wavelength of $800 \mathrm{~nm}$ and the power of $\sim 4 \mathrm{~mW}$ were selected for the study. Guided by literature data [13], detection of the SHG signal was performed with a spectral selection in the $371-415 \mathrm{~nm}$ wavelength range. The average value of SHG signal intensity and the SHG signal distribution concurrence (coherence) were selected as the parameters to use for quantitative evaluation of the condition of the collagen fibers, in particular, assessing the degree of integrity/ disorganization, form and their packing density.

The value of the average intensity reflects the relative number of collagen fibers in the field of view. The coherence is directly proportional to the degree of order of collagen fibers packing and therefore represents the isotropic properties of the image. It is determined as the ratio of the difference and sum of the maximum and minimum eigenvalues of the $2 \times 2$ matrix, obtained from the partial derivatives in the horizontal and vertical directions of the image. The value of the coherence parameter is limited in range from 0 to 1 , where 1 corresponds to highly oriented structures, and $0-$ to isotropic areas [24]. For evaluation of the average value of the SHG signal intensity from the collagen in the selected areas, the standard function for average 
value calculation provided by ImageJ software $(\mathrm{NIH}$, USA) was used [25]. For the assessment of collagen fiber orientation, a plugin for structural tensor analysis of ImageJ based on the OrientationJ program (NIH, USA) was used.

The parameters of average intensity and the coherence of distribution were calculated by means of averaging the values obtained from the ROI of the test sample. The ROI was chosen on the basis of the histological analysis and contained areas of typical tissue structure.

Atomic force microscopy. Histological sections mounted on glass slides were studied using the AFM method in air, in a semi-contact measurement mode to obtain topographic and phase images. AFM images were acquired with a Solver P47 microscope (NT-MDT, Russia), using a Solver scanner and TESP probes (Bruker, USA) with a nominal spring constant of $42 \mathrm{~N} / \mathrm{m}$, nominal resonant frequency of $320 \mathrm{kHz}$ and a nominal radius of tip curvature of $8 \mathrm{~nm}$. Overview images with the size of $14 \times 14 \mu \mathrm{m}$ were obtained from different areas of a tissue section using the sample orientations based on the corresponding tissue images from the optical microscope, combined with AFM, as well as from the photographs of each histological specimen. Such overview images allowed detection of topography of collagen fibers and their bundles.

In addition, high resolution topography and phase images of the $6 \times 6$ and $3 \times 3 \mu \mathrm{m}$ sizes were obtained. These high resolution images allowed detection of collagen fibrils' packing and characterization of individual fibrils.

The parameters of flicker-noise spectroscopy (FNS), obtained on the basis of the digitized AFM data, were used as a quantitative measure of the extracellular matrix ultrastructure. Initially, AFM images of the connective tissue sections in $14 \times 14 \mu \mathrm{m}$ windows were obtained, then structural fragments to be observed in $6 \times 6$ and $3 \times 3 \mu \mathrm{m}$ windows were selected from this area, since, at a higher resolution, the fibrils can be seen. The FNS parameters of the structure were determined for all the images obtained in such windows (typically, there were 5-8 images) [26]. It has previously been demonstrated [6] that, out of the entered 3D parameters of the structure in the specified range of spatial dimensions, the following ones are most sensitive to pathological changes in the tissue: parameter $\sigma$, which is a measure of the jump-like irregularities of the chaotic surface profiles against the low frequency - baseline it is determined as a "stepwiseness factor" of the chaotic component of the profile; and parameter $\mathrm{S}\left(\mathrm{L}_{0}{ }^{-1}\right)$, which is a measure of the spike-like irregularities of the profile being observed - it is determined as the "spikiness factor" of the surface structure. These two parameters were considered as the fundamental ones for the FNS parametrization of the bladder tissue AFM images.

ROI selection for quantitative assessment of the images. The ROls for quantitative assessment of the images from CP OCT, SHG and AFM were selected manually, with a particular size for each imaging technique. The ROls were located at two depths from the tissue surface and included only connective tissue (blood vessels and muscle fibers were excluded).

For the CP OCT images, two ROls were selected with dimensions of $200 \times 2,000$ and $300 \times 2,000 \mu \mathrm{m}$, at depths of $100-300 \mu \mathrm{m}$ corresponding to the mucous membrane where the inflammation process initially develops and the tumor cell invasion begins; and of 300-600 $\mu \mathrm{m}$ corresponding to the submucous layer, from which it is possible to assess the extent of the previous inflammation as well as the condition of the post-operative scar.

For SHG images, two ROls were also selected, with dimensions of $60 \times 60 \mu \mathrm{m}$, at depths of $100-300 \mu \mathrm{m}$ and 300-600 $\mu \mathrm{m}$, providing the opportunity to compare the results with the CP OCT data.

For AFM images, one ROI for each was selected with dimensions of $6 \times 6 \mu \mathrm{m}$ directly under the urothelium at a depth of up to $300 \mu \mathrm{m}$ (the region where maximally expressed morphological changes are observed).

Manual selection of the ROls was used for the focused analysis of changes in the connective tissue, where (primarily) the pathological process takes place (inflammation, tumor cell invasion). In the future, it will be helpful to advance in the direction of automated differential diagnostics of the bladder pathologies according to the characteristics of the collagen fibers state.

Statistical processing. The results were analyzed using Statistica 10.0 set (StatSoft, Inc., USA) and Prism 6 (GraphPad Software, USA). Average values (M) of the measured parameters and the average standard deviations $( \pm S D)$ were determined. The significance of the differences between the groups was calculated using the Mann-Whitney non-parametric test. For the determination of correlation ( $r$ ) Spearman's rank correlation coefficient was used. In all cases, the difference was considered to be significant at a significance value $p<0.05$.

Results and Discussion

Visual assessment of the collagen state in bladder pathologies, using CP OCT, SHG microscopy, AFM and histological methods. We had previously used CP OCT, SHG microscopy and histological methods on a stand-alone basis for the assessment of content, spatial and structural organization of the collagen fibers in the bladder wall [22]. However this is the first time when AFM has been used for the analysis of bladder connective tissue. In this study we performed a comparative assessment of the collagen condition by using these methods in four groups, described above (Figure 1).

In the case of mild inflammation (group 1, control) the CP OCT image (Figure 1 (a)) in co-polarization (upper part of the image) visually has a layered structure: the layer with a high signal level corresponds 
Mild inflammation of the bladder wall (group 1)
Severe inflammation of the bladder wall (group 2)
Poorly differentiated urothelial invasive bladder cancer (group 3)
Bladder cancer recurrence in the area

of the surgical scar (group 4)
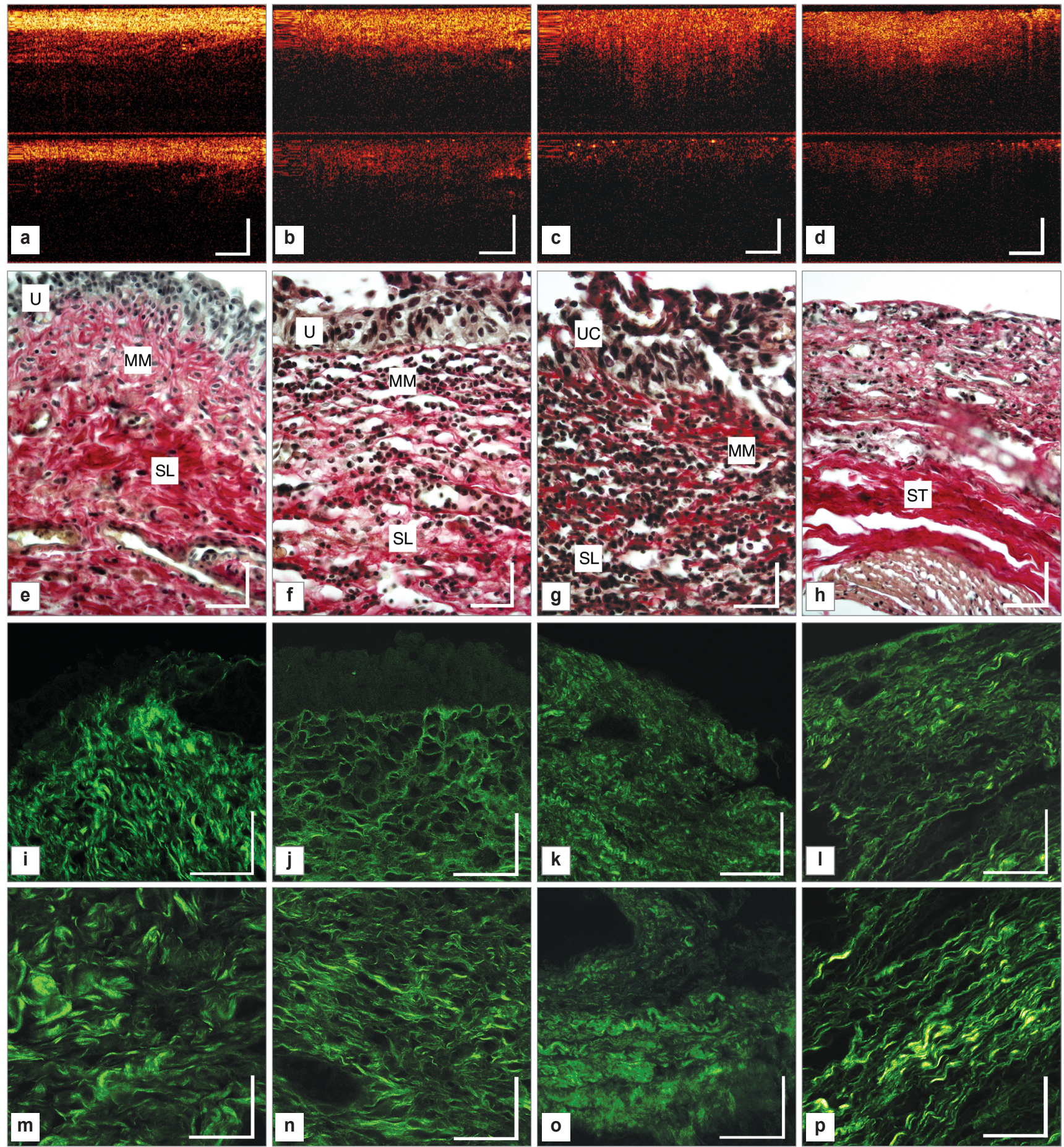

Figure 1. Collagen visualization of bladder pathologies by different imaging modalities: CP OCT image of the bladder wall (first row); corresponding histological specimen, stained by Van Gieson (second row); SHG images of the mucous membrane (third row); SHG images of the submucous layer (fourth row); topographic AFM images of the mucous membrane (fifth row); phase AFM images of the mucous membrane (sixth row). In the CP OCT images the upper part shows the image in copolarization; the lower part - in cross-polarization. Vertical and horizontal bars: $500 \mu \mathrm{m}$ for the CP OCT images; $50 \mu \mathrm{m}$ for the SHG and histological images; $1 \mu \mathrm{m}$ for the AFM images. Here: $\mathrm{U}$, urothelium; MM, mucous membrane; SL, submucous layer; UC, urothelial carcinoma; ST, scar tissue; for symbols (a)-(x) - see the text 

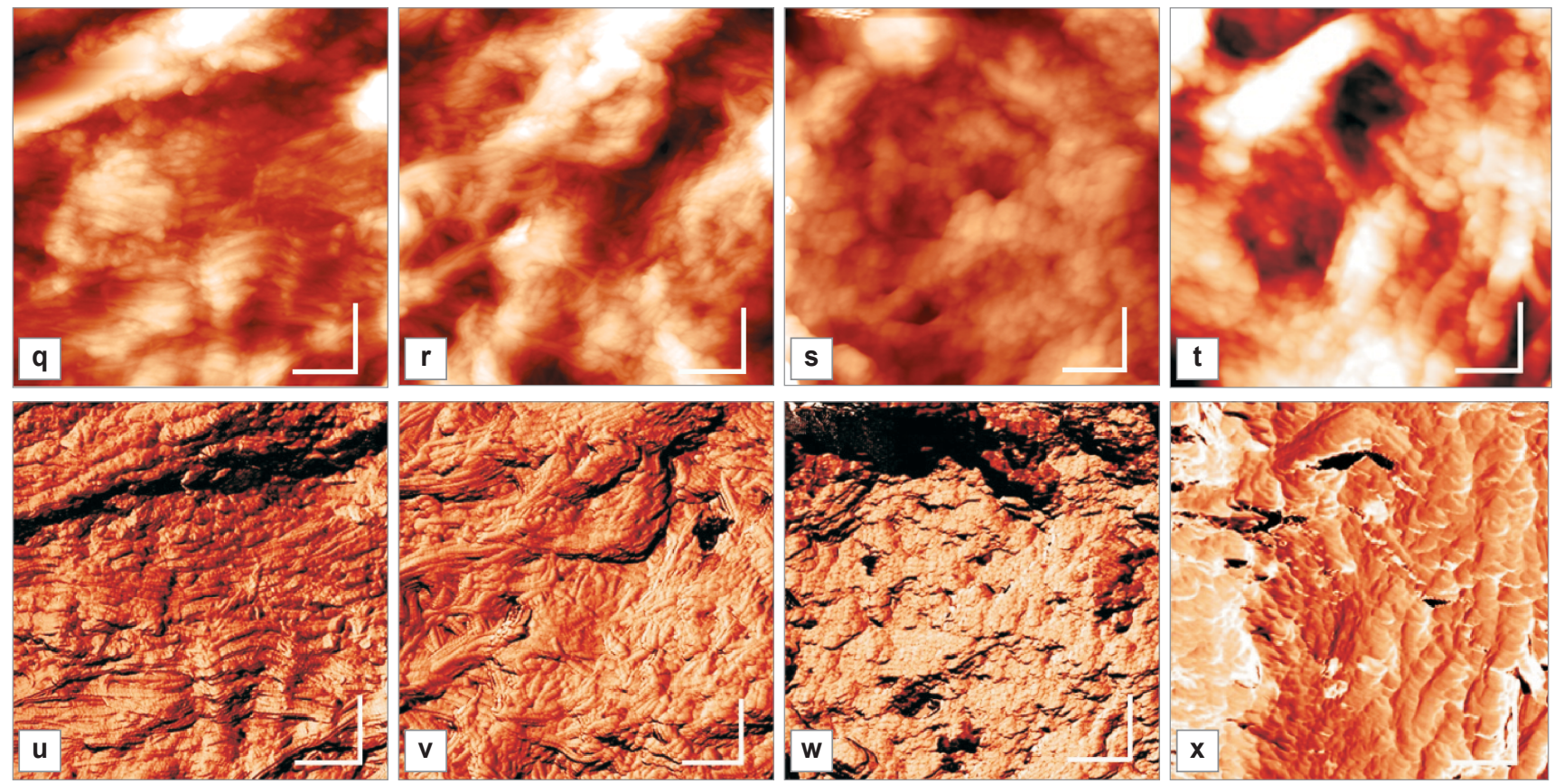

Figure 1 continued

to the bladder mucous and submucous membranes, while the underlying layer, with a lower signal level corresponds to the muscular layer. In cross-polarization (lower part of the image) a high level OCT signal can be observed, indicating the relatively ordered, closelypacked arrangement of collagen fibers in the bladder mucous and submucous membranes. It is also evident in the histological specimen with Van Gieson staining (Figure 1 (e)).

In the SHG images the collagen fibers are well visualized, they have a waved form and are about 2-3 $\mu \mathrm{m}$ thick. Overall, we may conclude on the existence of a preferred orientation (Figure 1 (i)), while deeper, in the submucous layer, fiber bundles with a thickness of 12-17 $\mu \mathrm{m}$ with the curved structure prevail which does not indicate the existence of a preferred direction (Figure $1(\mathrm{~m})$ ).

Collagen fibers and bundles, some partially denuded and others, partially covered with an "amorphous" (structureless) material, can be seen in the overview AFM images. Closely packed, short collagen fibrils with a parallel layering (Figure 1 (q)) can be clearly observed inside the collagen fibers. Collagen fibrils are characterized by their uniform thickness and easily distinguishable D-periods, as seen in the AFM phase images, obtained at a high resolution (Figure $1(\mathrm{u})$ ).

In the case of severe inflammation (group 2) the signal level in the CP OCT image (Figure 1 (b), upper part of the image) is noticeably lower in comparison to that in the case of mild inflammation, due to the edema at the mucous membrane and tissue infiltration by the inflammatory cells (lymphocytes) (Figure 1 (f)). Signal attenuation takes place at approximately the same depth across the whole image. In cross-polarization (Figure 1 (b), lower part of the image) the signal level is significantly lower, which is an indicative of a decrease in the polarization properties of the extracellular matrix due to thinning of the mucous membrane collagen fibers and significant mucoid swelling in the submucous layer. This was confirmed by the high resolution microscopy (SHG and $\mathrm{AFM}$ ).

The SHG signal from the collagen fibers, in this case, has lower intensity (Figure $1(\mathrm{j}),(\mathrm{n}))$ as compared to the control group (See Figure 1 (i), (m)). Collagen fibers in the mucous membrane are thinned (average thickness is $1-1.5 \mu \mathrm{m})$, and they have indistinct boundaries, which is an indicative of damage to their structure. Attention should also be paid to the uneven distribution of the fibers throughout the tissue. The fibers are located loosely indicating no preferred direction of orientation (See Figure 1 (j)). In the submucous layer, the collagen fibers are thickened due to mucoid swelling and are arranged more tightly (See Figure $1(n)$ ), with characteristics close to those of the samples from group 1 at this depth.

AFM images of severe inflammation in the bladder mucosa reveal that the packing of the matrix collagen fibers does not change, however, the packing of collagen fibrils within the fibers has changed - densely interweaved texture of chaotically oriented fibrils, forming a "net" can be observed (Figure 1 (r), (v)).

In the case of poorly differentiated invasive urothelial carcinoma (group 3) the CP OCT image in copolarization (Figure 1 (c), upper part of the image) is structureless and the OCT signal level is low. Particularly note the inhomogeneous distribution of the signal along 
the transverse coordinate and pronounced vertical lines in the image structure. This feature of the OCT signal is conditioned by the infiltrative growth of cancer. According to the histological specimen, stained according to Van Gieson (Figure $1(\mathrm{~g})$ ), it is evident that the majority of the tumor cells are located at the mucous membrane, disturbing the structure of the connective tissue. Tumor cell clusters can also be observed in the submucous layer, but here inflammatory infiltration prevails. In crosspolarization (Figure 1 (c), lower part of the image) the signal is either very weak or there is no signal at all. These features of CP OCT images of invasive bladder cancer have been previously described by our group [9].

It can be seen in the SHG images that depending on the distance from the tissue surface the collagen fibers exhibit different states: at the depth of the mucous membrane the collagen fibers are either destroyed or fragmented (Figure $1(\mathrm{k})$ ) due to the invasive growth of the urothelial carcinoma; in the submucous layer aggregation of collagen fibers into bundles can be observed, and, in some parts, such bundles are thickened with the borders of the bundles and fibers being indistinct (Figure 1 (o)) due to the long duration of the current inflammation.

In the course of AFM analysis the following trend was revealed: in the overview images demonstrating the microstructure of the extracellular matrix, collagen fibers do not have sharp contours and are entirely covered with a non-fibrous amorphous material, which (at a higher resolution) (Figure $1(\mathrm{~s}),(\mathrm{w}))$ is presented in the form of "beads" of another protein (protein-based proteoglycan complexes), which completely cover collagen fibrils. Consequently, according to the AFM data, invasive cancer changes the ratio between the collagen fibers and the non-fibrous components of the matrix: the fraction of collagen fibers in the matrix is decreased, which obviously correlates with the low level of crossscattering in the CP OCT image.

In the case of recurrence of poorly differentiated invasive urothelial carcinoma in scar tissue (group 4), the CP OCT image in co-polarization is also structureless at the mucous membrane (similar to the case of invasive cancer) (Figure 1 (d), upper part of the image), however, the OCT signal level, in general, is higher due to fibrosis (Figure $1(\mathrm{~h})$ ). Along the transverse coordinate the signal is homogenous (overall), with the vertical lines, although pronounced weak as compared to previous group. However, in this case the image in cross-polarization is not typical for invasive poorly differentiated cancer. The absence of an OCT signal at the surface reflects the existence of the tumor, which does not produce any cross-scattering. At larger depths an OCT signal of varying intensity that is non-uniform along the transverse coordinate (Figure 1 (d), lower part of the image) is observed. The existence of areas with either high or low level of OCT signals can be explained morphologically by the presence of regions with coherently laid fibers and bundles at the surgical scar as well as areas of disorganized collagen fibers under the influence of the cathepsins and proteinases released by the tumor cells in the areas of intense infiltration of the scar tissue by the tumor cells (See Figure $1(h)$ ).

Non-uniform in-depth attenuation of the OCT signal in cross-polarization in the case of cancer in the scar area is confirmed by the difference in the SHG signals at different depths. Near the surface, in the area of intense infiltration of the scar tissue by the cancer cells (Figure 1 (I)), disorganization of the collagen is observed, and the boundaries of the bundles and the fibers are indistinct rather than sharp. Deeper, at the level of the submucous layer, there are areas where the scar tissue structure is preserved: collagen fiber bundles with sharp boundaries prevail, and a preferred orientation is evident; with high SHG signal intensity from the fiber is (Figure $1(p)$ ).

In the corresponding overview AFM images there are collagen fiber bundles that are partially denuded and some that are partially covered with amorphous material. Topography (Figure $1(\mathrm{t})$ ) and phase imaging (Figure 1 (x)) visualize a coherent layering of the fibrils and the typical banding (D-period) can be observed. These findings indicate the processes of the fibrous (scar) tissue formation in the area of the surgical wound.

Consequently, a visual comparative assessment of the CP OCT images and images from high resolution microscopy illustrates a high degree of data correspondence. This allows justification of the concept that the character of the OCT signal in CP OCT images of the bladder in the case of these pathologies matches changes affecting the collagen fibers at various levels of organization. However, visual assessment of the images is demanding and requires detailed knowledge that is rarely available in actual clinical practice. In this respect quantitative assessment of the images is reasonable.

Quantitative assessment of CP OCT images. In this study we performed a quantitative assessment of cross-scattering by generating depolarization factor maps from the CP OCT images. Color encoding of the depolarization factor values in the range from 0 to 0.3 facilitated the visual assessment of the cross-scattering properties of the connective tissue stroma basing on $\mathrm{CP}$ OCT images consisting in per-pixel calculation of the ratio of the signal levels in cross- and co-polarization. Selection of ROls in depolarization factor maps at different depths from the tissue surface with calculation of IDF makes the assessment of CP OCT images objective.

Depolarization factor maps typical for the four groups of bladder pathologies that were studied are presented in Figure 2, while the IDF values (depolarization factors averaged over ROls) are shown in Figure 3 . The area marked in Figure 2 with a grey rectangle morphologically corresponds to the bladder mucous membrane; while the area marked with an orange rectangle corresponds to the submucous layer.

In the group with mild inflammation (group 1) the depolarization factor value at the depth of the mucous 


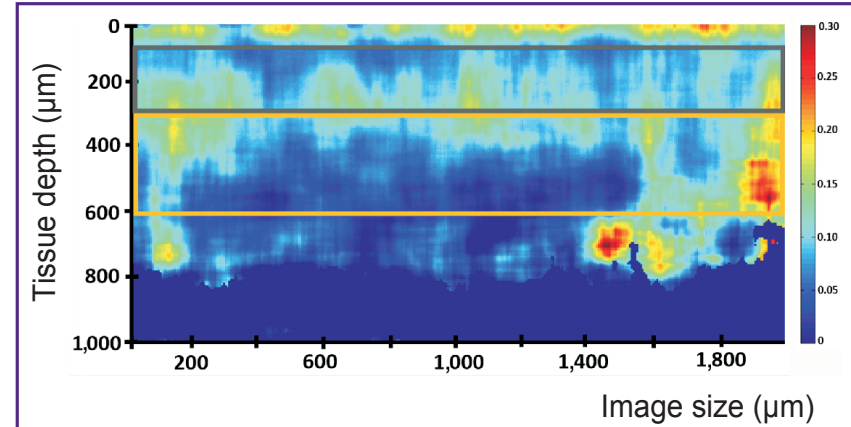

a

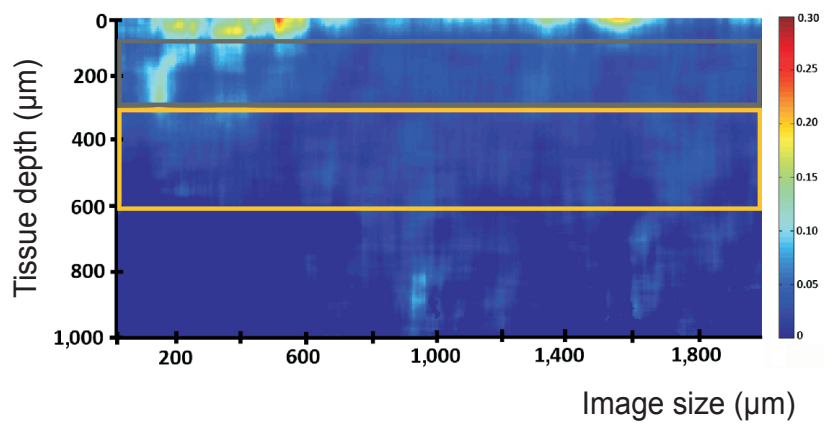

C

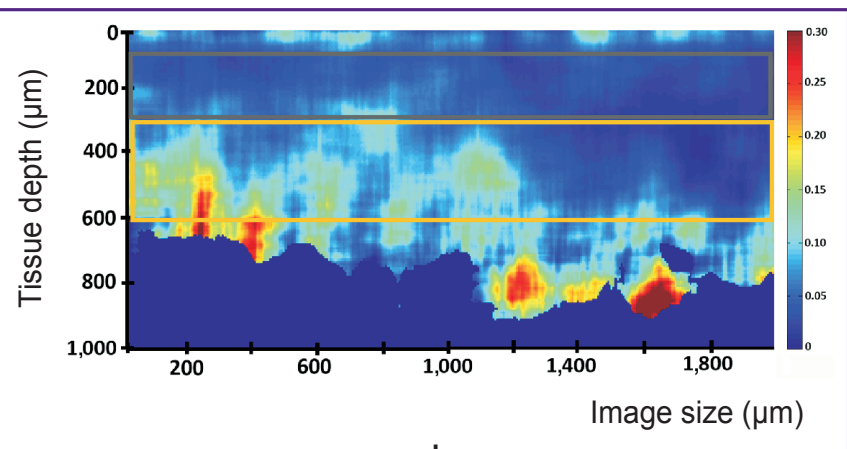

b

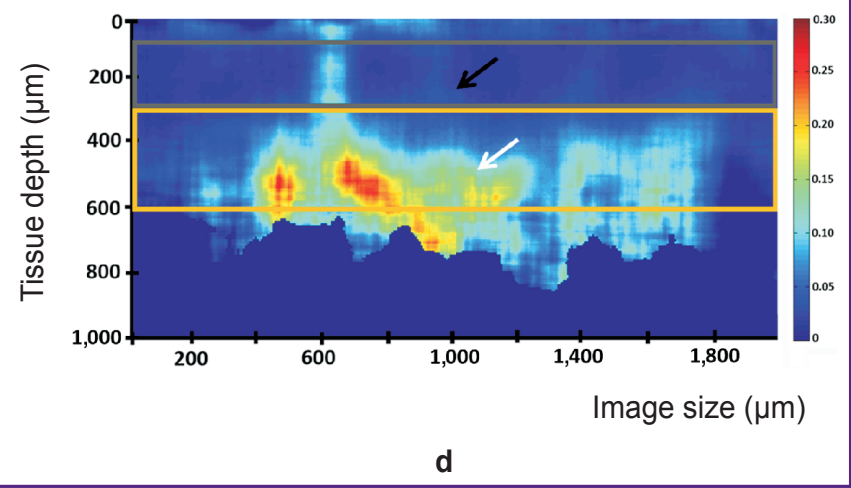

Figure 2. Depolarization factor maps for mild (a) and severe (b) inflammation of the bladder wall; at poorly differentiated invasive urothelial carcinoma (c) and urothelial carcinoma recurrence in the area of the post-operative scar (d); black pointer - tumor area; white pointer — scar area; axis tick labels are specified in $\mu \mathrm{m}$; the jet colormap represent the color encoding of the IDF values

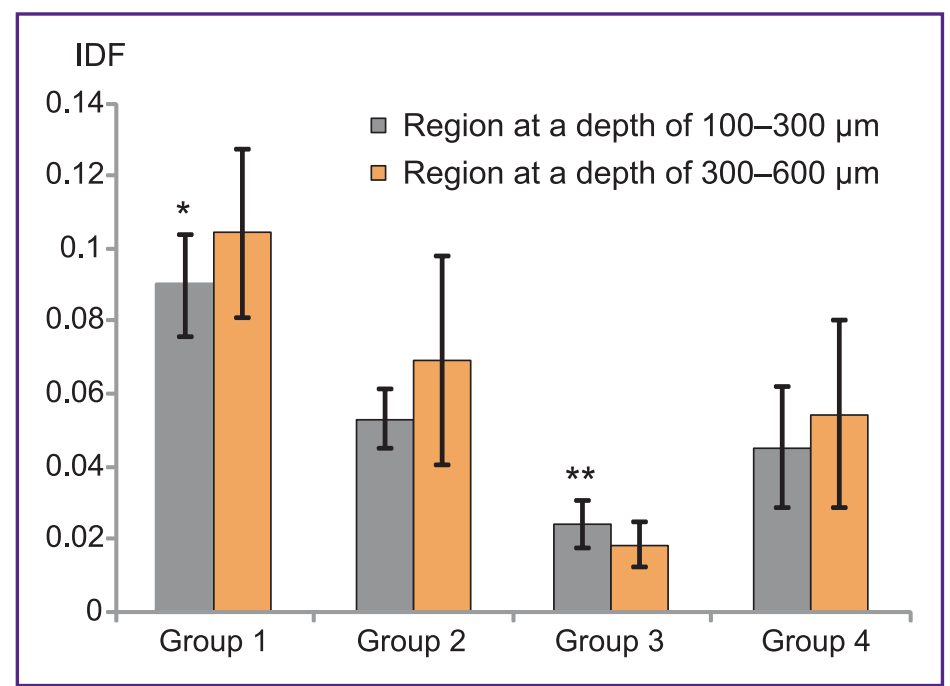

Figure 3. Histogram of the IDF value distribution for mild (group 1) and severe (group 2) inflammation of the bladder wall; for poorly differentiated invasive urothelial carcinoma (group 3) and urothelial carcinoma recurrence in the area of the post-operative scar (group 4). * Statistically significant difference between the control group and all the other groups of pathologies, $p<0.05$ (Mann-Whitney criterium); ** between urothelial carcinoma and the other pathologies, $p<0.05$ (Mann-Whitney criterium) membrane is higher than in the case of the other pathologies (groups 2-4) (Figure 2 (a)-(d), grey rectangle; Figure 3, grey columns), which matches the observations (obtained in the course of SHG, AFM and histological examination) concerning the preservation of the structure of the majority of the collagen fibers in this condition (See Figure 1 (i), (m)) and at this depth.

While comparing the depolarization factor values in the maps and the calculated IDF values for the ROI at a depth of 300-600 $\mu \mathrm{m}$, an actual decrease of this parameter is observed only in the group with urothelial invasive carcinoma (group 3 ). In the other groups (1, 2 and 4) the IDF values remain high, although statistically significant differences are not achieved (Figure 2 (a)(d), orange rectangle; Figure 3 , orange columns). This is also consistent with the results of the parallel examinations using high resolution methods. Only in the case of invasive urothelial carcinoma developing against 
particularly severe inflammation the collagen fibers and fibrils at this depth become disorganized and fragmented to a noticeable extent. In cases of mild and severe inflammation, as well as in cases of the recurrence of urothelial carcinoma in the area of the surgical scar at the level of the submucous layer, the collagen fibers are predominantly in a condition of mucoid swelling, becoming thickened and densely arranged. This results in efficient cross-scattering.

It is important that the results of the IDF calculation demonstrate statistically significant differences between the groups with severe inflammation and those with urothelial carcinoma (See Figure 3, $p<0.05$ ) confirming the efficacy of the diagnostic use of CP OCT in these situations.

Thus, a statistically significant decrease in the IDF values derived from the CP OCT images of bladder cancer against a background of chronic inflammation and carcinoma recurrence in the area of the surgical scar confirms the significant disorganization of the collagen fibers (responsible for the polarization properties of the tissue) in the case of carcinoma invasion into the connective tissue. Consequently, the IDF may be used to detect (with a high level of reliability) urothelial carcinoma against a background of severe inflammation, as well as carcinoma recurrence in the area of the surgical scar.

Quantitative assessment of SHG images. Two parameters are proposed for quantitative assessment of the collagen state from the SHG images: the average value of the signal intensity, and the coherence of its distribution. Results for each of the four groups are demonstrated in Figure 4. It can be seen that at the different depths from the bladder surface these parameters have different characteristic values, which is an indicative of the heterogeneity of the studied tissues.

In particular, note that the coherence of the SHG signal distribution in the bladder is low, amounting about 0.125 , indicating the absence of a preferred direction of the extracellular matrix collagen fibers in the bladder wall. The highest value of average intensity and coherence in the SHG signal can be observed in the case of mild inflammation of the bladder wall directly under the urothelium (Figure 4, grey columns). Statistically significant differences in these two parameters exist between the control group and the groups with severe inflammation, poorly differentiated carcinoma, and carcinoma in the area of the surgical scar (See Figure 4, p<0.05). However, at greater depth $(300-600 \mu \mathrm{m})$ a significant decrease of these parameters of the SHG signal can be observed in the control group and this is an indicative of the structural inhomogeneity and multidirectionality of the packing of the collagen fibers at the level of the submucous layer. This seems to be due to the intrusion of the muscle fibers among the connective tissue fibers at this depth.

The lowest values of these two parameters of the SHG signal at a depth of $100-300 \mu \mathrm{m}$ from the tissue surface are found in the cases of both severe inflammation and poorly differentiated urothelial carcinoma being an indicative of the absence of a preferred orientation of the collagen fibers in the subsurface area in these

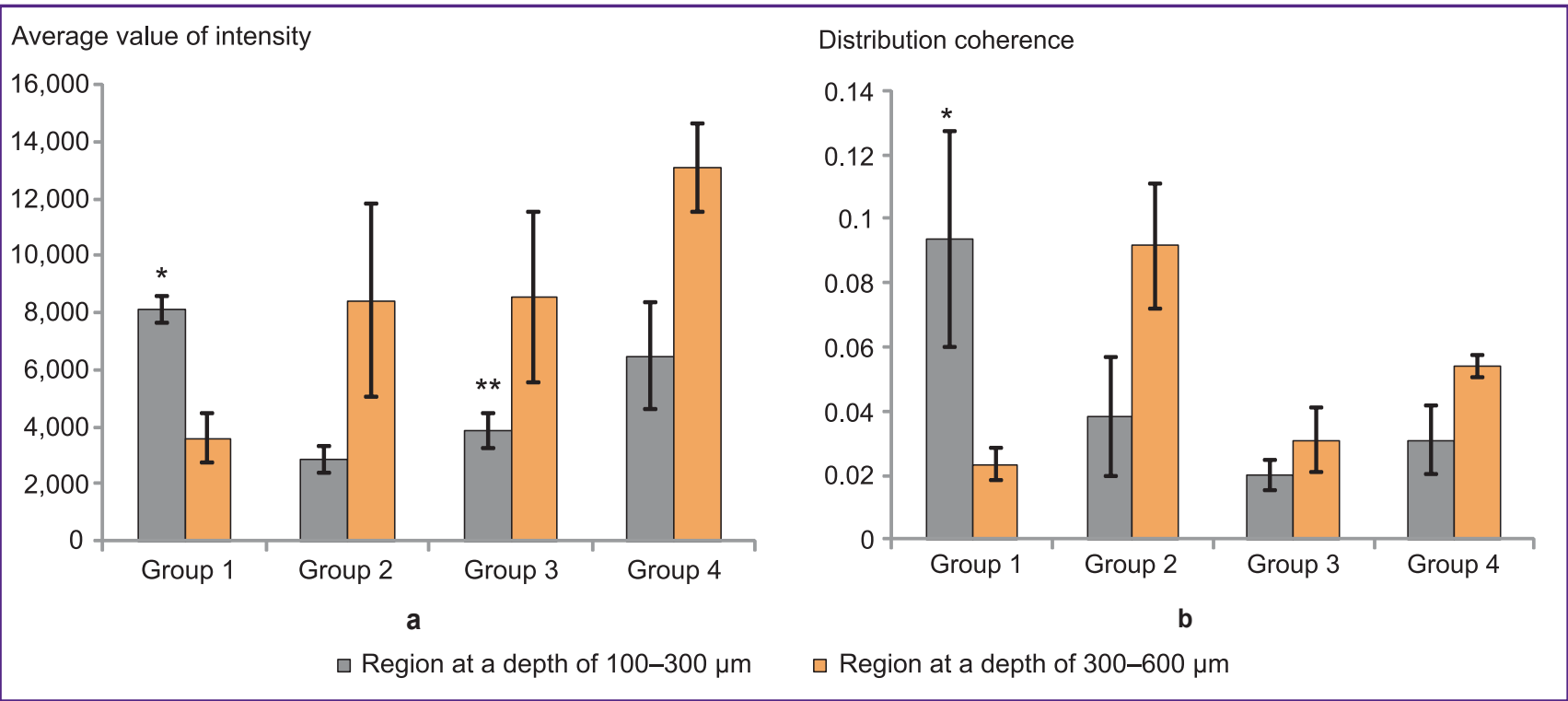

Figure 4. Histograms of the average value of intensity (a) and the distribution coherence (b) of the SHG signal for mild (group 1) and severe (group 2) bladder wall inflammation; for poorly differentiated invasive urothelial carcinoma (group 3 ) and for urothelial carcinoma recurrence in the area of the post-operative scar (group 4). * Statistically significant difference of the SHG signal parameters in the mucous membrane, calculated at the same depth as in group 1 in comparison with the other pathologies, $p<0.05$ (Mann-Whitney criterium); ${ }^{* *}$ values of average intensity of the SHG signal in the mucous membrane in groups 2 and 3, $\mathrm{p}<0.05$ (Mann-Whitney criterium) 


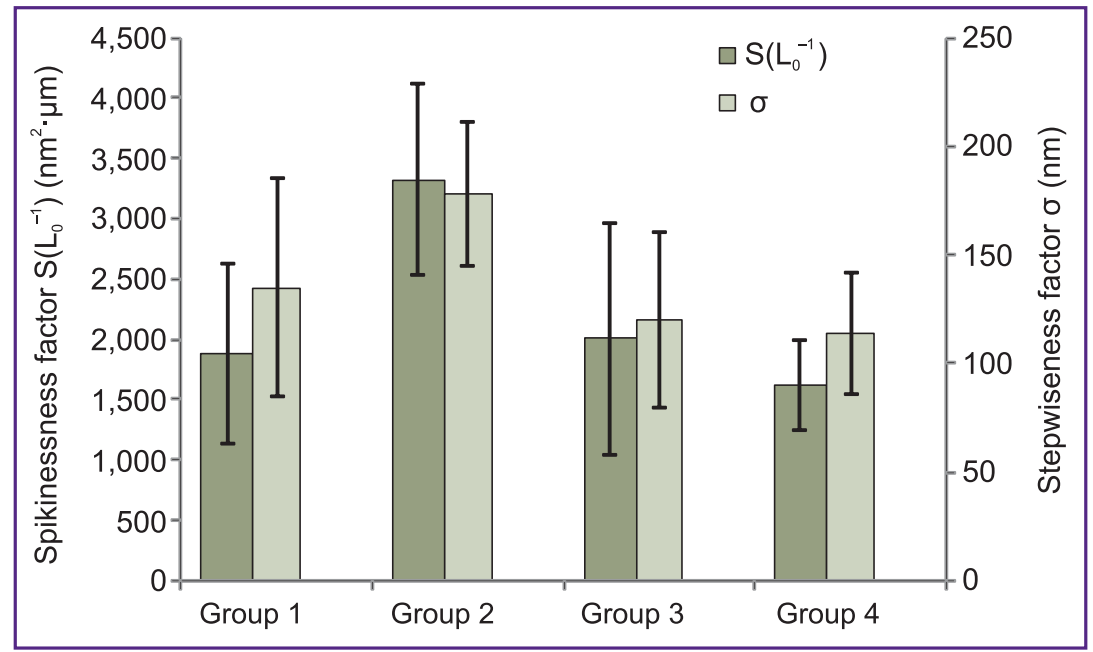

Figure 5. FNS parameters of the surface for AFM images of the bladder tissue at mild inflammation (group 1) and severe inflammation (group 2); at poorly differentiated urothelial carcinoma (group 3) and recurrence of carcinoma in the area of the post-operative scar (group 4)

cases. It is important to note that a statistically significant difference $(p<0.05)$ in the average values of the SHG signal between the severe inflammation and the urothelial carcinoma groups (Figure $4(a)$ ) is revealed. By contrast we were unable to find a consistent change of the average value of the SHG signal intensity with depth in the cases under study.

The values of coherence of the SHG signal distribution at the depth of the mucous membrane mirrored the IDF values, i.e. they were largest in the case of mild inflammation, and smallest in the case of carcinoma invasion (Figure 4 (b)). At a depth of 300-600 $\mu \mathrm{m}$ no trends in variation of the SHG signal coherence values or in the intensity values in the cases under study were revealed.

Thus, the proposed parameters, namely, average value of the SHG signal intensity and the coherence of its distribution, allow for assessment of the spatial location and organization of the collagen fibers in the bladder wall at different depths and in cases of different pathological processes.

For verification of the CP OCT diagnostic data, a correlation analysis with the SHG signal parameters in the selected ROls was performed. A highly statistically significant positive correlation between the IDF and the parameter of conformance of the SHG signal distribution from collagen at the level of the mucous membrane ( $r=0.867 ; p<0.001$; Spearman correlation) was revealed. This is an indicative of high degree of correlation between the cross-scattering level and collagen fibers packing density. This determines the structural disorganization of the fibers at a tissue level in the bladder subsurface region. However, a significant decrease in correlation can be observed between the specified parameters at the level of the submucous layer $(r=0.127 ; p=0.648$; Spearman correlation).
Between the values of the IDF and the parameter of the SHG intensity signal from the collagen at the level of mucous membrane, a low and statistically insignificant correlation was revealed $(r=0.464 ; p=0.081$; Spearman correlation). For the submucous layer no correlation between the specified parameters $\quad(r=-0.116 ; \quad p=0.682$; Spearman correlation) was found. Consequently, a correlation analysis of these quantitative parameters reveals only their unconditional relationship, while a visual assessment is sensitive to less evident relationships.

FNS parametrization of AFM images. The FNS parameters were obtained for the AFM images of the bladder extracellular matrix in all the situations being studied. In the cases of mild and severe inflammation, we observed significant variance of FNS parameters (groups 1 and 2, Figure 5), which is indicative of the extreme non-uniformity of the extracellular matrix in these cases. In the case of urothelial carcinoma of the bladder, the FNS parameters were either unchanged or decreased, in comparison with the control group, although the variance of the values was not significant (groups 3 and 4, Figure 5). It is obvious, that the observed trend for the tumor process reflects a smoothing of the surface topography, associated with the increase in the content of nonfibrous matrix components, covering the destroyed collagen structures. The correlation of these FNS parameters with the CP OCT and SHG parameters was not determined.

Discussion. These studies demonstrate the potential of $\mathrm{CP}$ OCT as an in vivo technique for assessing the state of collagen fibers that may be used for the reliable diagnosis of bladder cancer developing against a background of severe inflammation, as well as of carcinoma recurrence in the area of the post-operative scar.

Three modalities of high resolution technologies were used to provide visualization and a comprehensive assessment of the collagen fibers, the dominant component of connective tissue, and to trace the changes in its state in the bladder wall that occur in cases of considered pathologies. These approaches allowed for evaluation of the collagen fibers from the viewpoint of their integrity/disorganization, orientation, form and density by characterizing the condition of the collagen at different levels of its structural organization (from tissue to fibrillar) and to draw conclusions on the sensitivity of the OCT signal in cross-polarization to the changes in the condition of the collagen fibers. It has been demonstrated that the optical structures in the $\mathrm{CP}$ OCT images are visually well correlated with the results 
of traditional histopathology and with the assessment of collagen by means of SHG and AFM.

Quantitative processing of the images obtained by $\mathrm{CP}$ OCT and high resolution microscopy (SHG microscopy and AFM modes) allowed objective assessment of the changes in the spatial organization of the extracellular matrix at a multi-structural level for different stages of the inflammatory process and for invasive bladder cancer.

Results of the IDF calculations (evaluating the condition of the collagen fibers by the level of crossscattering) demonstrated the potential for its use in automated diagnoses of bladder pathology. The obvious decreases in the IDF value both in the cases of invasive bladder cancer developing against a background of chronic inflammation and cancer recurrence in the area of the post-operative scar confirm significant disorganization of the collagen fibers in the case of cancer invasion. Therefore, employment of IDF allows the detection (with high level of significance) of urothelial carcinoma against a background of severe inflammation, as well as carcinoma recurrence in the area of the postoperative scar $(p<0.05)$.

Study of the two SHG signal parameters, the average value of intensity and the coherence of its distribution, at the level of the mucous and submucous membranes of the bladder wall demonstrated that their lowest values directly under urothelium (mucous membrane) are found in cases of severe inflammation and poorly differentiated urothelial carcinoma. This is an indicative of the absence of a preferred orientation of the collagen fibers in the subsurface area in these situations. The statistically significant difference $(p<0.05)$ found for the average value of the SHG signal intensity in the groups with severe inflammation and with urothelial carcinoma is considered to be important. The values of SHG signal distribution coherence at the level of the mucous membrane mirrored the trends for IDF, i.e. they were greatest in the case of mild inflammation and lowest in the case of cancer invasion.

The IDF calculation provides significant information concerning the level of disorganization of the stromal collagen in the case of invasion correlating well with the data from nonlinear microscopy in SHG mode. The Spearman correlation coefficient for the IDF and SHG signal distribution coherence was $r=0.867 ; p<0.001$.

The character of the changes taking place in the bladder in the case of inflammation and cancer developing at its background is confirmed by the AFM method with the use of qualitative assessment of the FNS parameters. The involvement of all levels of collagen organization inevitably affects the surface structure. Significant variance of the FNS parameters in the case of inflammation (independently of its expression) is indicative of the extreme non-uniformity of the extracellular matrix in these cases. In the case of bladder tumors, the FNS parameters were slightly changed in comparison with the control group, although the variance of values was not significant. It is obvious, that the observed trend for the tumor process reflects smoothing of the surface topography, connected with an increase in the content of non-fibrous matrix components (protein-based proteoglycan complexes), covering the collagen structures.

The comprehensive quantitative assessment of $\mathrm{CP}$ OCT images and high resolution microscopy (SHG and AFM) in the case of inflammation and of bladder cancer enables to trace disorganization of the collagen at different levels: at a tissue level (decrease of crosspolarization back-scattering, which is quantitatively characterized by the IDF); at the fiber level (severe mucoid swelling and fragmentation in the SHG images quantitatively characterized by the average value of intensity and coherence of the SHG signal distribution); and at the fibrillary level (distortion of fibril layering, prevalence of interweaving of the net of fibrils, and presence of amorphous material seen in the AFM images, which can be quantitatively characterized by their FNS parameters).

By contrast to OCT, the use of SHG microscopy and AFM modes in clinical endoscopic practice is currently unavailable. In this study, methods of high resolution microscopy were considered as the means of verification of the changes in the microstructure of the bladder extracellular matrix in cases of inflammation and cancer which improved understanding of the features observed in CP OCT images.

Conclusion. Comparison of CP OCT images with the data from high resolution microscopic modalities (nonlinear microscopy in second harmonic generation mode and atomic force microscopy) demonstrated that CP OCT has the potential to become a powerful, real-time and minimally invasive tool for the diagnosis of bladder cancer against a background of severe inflammation in the area of the post-operative scar. The presented results should stimulate the further development of CP OCT technique and promote its active introduction into clinical practice.

Study Funding. The study was supported by the Russian Science Foundation, agreement No.14-1500538 (EVG, EBK, VVD, SLK, LBT, MYuK, GVB, NDG).

Conflicts of Interest. The authors declare no conflicts of interest.

\section{References}

1. Leggett C.L., Gorospe E.C. Application of confocal laser endomicroscopy in the diagnosis and management of Barrett's esophagus. Ann Gastroenterol 2014; 27(3): 193-199.

2. Wessels R., De Bruin D.M., Faber D.J., Van Leeuwen T.G., Van Beurden M., Ruers T.J. Optical biopsy of epithelial cancers by optical coherence tomography (OCT). Lasers Med Sci 2014; 29(3): 1297-1305, https://doi. org/10.1007/s10103-013-1291-8.

3. Zhou C., Tsai T.H., Lee H.C., Kirtane T., Figueiredo M., Tao Y.K., Ahsen O.O., Adler D.C., Schmitt J.M., Huang Q., Fujimoto J.G., Mashimo H. Characterization of buried glands before and after radiofrequency ablation by using 
3-dimensional optical coherence tomography (with videos). Gastrointest Endosc 2012; 76(1): 32-40, https://doi. org/10.1016/j.gie.2012.02.003.

4. Pande P., Shrestha S., Park J., Gimenez-Conti I., Brandon J., Applegate B.E., Jo J.A. Automated analysis of multimodal fluorescence lifetime imaging and optical coherence tomography data for the diagnosis of oral cancer in the hamster cheek pouch model. Biomed Opt Express 2016; 7(5): 2000-2015, https://doi.org/10.1364/boe.7.002000.

5. Ren H., Yuan Z., Waltzer W., Shroyer K., Pan Y. Enhancing detection of bladder carcinoma in situ by 3-dimensional optical coherence tomography. J Urol 2010; 184(4): 1499-1506, https://doi.org/10.1016/j.juro.2010.05.087.

6. Hale N.E., Deem S. Advances in cystoscopic surveillance of superficial bladder cancer: detection of the invisible tumor. Medical Instrumentation 2013; 1(1): 6, https:// doi.org/10.7243/2052-6962-1-6.

7. Burns J.A., Kim K.H., deBoer J.F., Anderson R.R., Zeitels S.M. Polarization-sensitive optical coherence tomography imaging of benign and malignant laryngeal lesions: an in vivo study. Otolaryngol Head Neck Surg 2011; 145(1): 91-99, https://doi.org/10.1177/0194599811403078.

8. Villiger M., Lorenser D., McLaughlin R.A., Quirk B.C., Kirk R.W., Bouma B.E., Sampson D.D. Deep tissue volume imaging of birefringence through fibre-optic needle probes for the delineation of breast tumour. Sci Rep 2016; 6: 28771, https://doi.org/10.1038/srep28771.

9. Gladkova N., Kiseleva E., Streltsova O., Prodanets N., Snopova L., Karabut M., Gubarkova E., Zagaynova E. Combined use of fluorescence cystoscopy and crosspolarization OCT for diagnosis of bladder cancer and correlation with immunohistochemical markers. J Biophotonics 2013; 6(9): 687-698, https://doi.org/10.1002/jbio.201200105.

10. Bredfeldt J.S., Liu Y., Pehlke C.A., Conklin M.W., Szulczewski J.M., Inman D.R., Keely P.J., Nowak R.D., Mackie T.R., Eliceiri K.W. Computational segmentation of collagen fibers from second-harmonic generation images of breast cancer. J Biomed Opt 2014; 19(1): 016007, https://doi. org/10.1117/1.jbo.19.1.016007.

11. König K., Raphael A.P., Lin L., Grice J.E., Soyer H.P., Breunig H.G., Roberts M.S., Prow T.W. Applications of multiphoton tomographs and femtosecond laser nanoprocessing microscopes in drug delivery research. Adv Drug Deliv Rev 2011; 63(4-5): 388-404, https://doi. org/10.1016/j.addr.2011.03.002.

12. Cicchi R., Kapsokalyvas D., Troiano M., Campolmi P., Morini C., Massi D., Cannarozzo G., Lotti T., Pavone F.S. In vivo non-invasive monitoring of collagen remodelling by two-photon microscopy after micro-ablative fractional laser resurfacing. Biophotonics 2014; 7(11-12): 914-925, https:// doi.org/10.1002/jbio.201300124.

13. Cicchi R., Matthäus C. Meyer T., Lattermann A., Dietzek B. Brehm B.R., Popp J., Pavone F.S. Characterization of collagen and cholesterol deposition in atherosclerotic arterial tissue using non-linear microscopy. J Biophotonics 2014; 7(12): 135-143, https://doi.org/10.1002/jbio.201300055.

14. Campagnola R. Second harmonic generation imaging microscopy: applications to diseases diagnostics. Anal Chem 2011; 83(9): 3224-3231, https://doi.org/10.1021/ac1032325.

15. Seidenari S., Arginelli F., Dunsby C., French R., $_{\text {, }}$ König K., Magnoni C., Manfredini M., Talbot C., Ponti G. Multiphoton laser tomography and fluorescence lifetime imaging of basal cell carcinoma: morphologic features for noninvasive diagnostics. Exp Dermatol 2012; 21(11): 831-836, https://doi.org/10.1111/j.1600-0625.2012.01554.x.

16. Stolz M., Gottardi R., Raiteri R., Miot S., Martin I., Imer R., Staufer U., Raducanu A., Duggelin M., Baschong W., Daniels A.U., Friederich N.F., Aszodi A., Aebi U. Early detection of aging cartilage and osteoarthritis in mice and patient samples using atomic force microscopy. Nat Nanotechnol 2009; 4(3): 186-192, https://doi.org/10.1038/ nnano.2008.410.

17. Lekka M., Laidler P., Gil D., Lekki J., Stachura Z., Hrynkiewicz A.Z. Elasticity of normal and cancerous human bladder cells studied by scanning force microscopy. Eur Biophys J 1999; 28(4): 312-316, https://doi.org/10.1007/ s002490050213.

18. Timashev P.S., Kotova S.L., Belkova G.V., Gubar'kova E.V., Timofeeva L.B., Gladkova N.D., Solovieva A.B. Atomic force microscopy study of atherosclerosis progression in arterial walls. Microsc Microanal 2016; 22(2): 311-325, https://doi.org/10.1017/S1431927616000039.

19. Gelikonov V., Gelikonov G., Shilyagin P. Linearwavenumber spectrometer for high-speed spectral-domain optical coherence tomography. Opt Spectrosc 2009; 106: 459465, https://doi.org/10.1134/s0030400x09030242.

20. Gubarkova E.V., Dudenkova V.V., Feldchtein F.I., Timofeeva L.B., Kiseleva E.B., Kuznetsov S.S., Shakhov B.E., Moiseev A.A., Gelikonov V.M., Gelikonov G.V., Vitkin A., Gladkova N.D. Multi-modal optical imaging characterization of atherosclerotic plaques. J Biophotonics 2016; 9(10): 10091020, https://doi.org/10.1002/jbio.201500223.

21. Moiseev A.A., Gelikonov G.V., Terpelov D.A., Shilyagin P.A., Gelikonov V.M. Noniterative method of reconstruction optical coherence tomography images with improved lateral resolution in semitransparent media. Laser Physics Letters 2013; 10(12): 125601, https://doi. org/10.1088/1612-2011/10/12/125601.

22. Kiseleva E., Kirillin M., Feldchtein F., Vitkin A., Sergeeva E., Zagaynova E., Streltzova O., Shakhov B., Gubarkova E., Gladkova N. Differential diagnosis of human bladder mucosa pathologies in vivo with cross-polarization optical coherence tomography. Biomed Opt Express 2015; 6(4): 1464-1476, https://doi.org/10.1364/BOE.6.001464.

23. Gubarkova E.V., Kirillin M.Yu., Sergeeva E.A., Kiseleva E.B., Snopova L.B., Prodanets N.N., Sharabrin E.G., Shakhov E.B., Nemirova S.V., Gladkova N.D. Crosspolarization optical coherence tomography in evaluation of atherosclerotic plaque structure. Sovremennye tehnologii $v$ medicine 2013; 5(4): 45-55.

24. Rezakhaniha R., Agianniotis A., Schrauwen J.T.C., Griffa A., Sage D., Bouten C.V.C., van de Vosse F.N., Unser M., Stergiopulos N. Experimental investigation of collagen waviness and orientation in the arterial adventitia using confocal laser scanning microscopy. Biomech Model Mechanobiol 2012; 11: 461, https://doi.org/10.1007/s10237-011-0325-z.

25. Rasband W.S. ImageJ, U. S. National Institutes of Health, Bethesda, Maryland, USA (1997-2008). Image J Image Processing and Analysis in Java. URL: http://rsb.info.nih.gov/ij/.

26. Mirsaidov U., Timashev S.F., Polyakov Y.S., Misurkin P.I., Musaev I., Polyakov S.V. Analytical method for parameterizing the random profile components of nanosurfaces imaged by atomic force microscopy. Analyst 2011; 136(3): 570-576, https://doi.org/10.1039/c0an00498g. 